\title{
Magnetic characterization of self-organized ErAs clusters using telegraph noise spectroscopy
}

\author{
F. Coppinger* \\ High Magnetic Field Laboratory, Max-Planck-Institut für Festkörperforschung and Centre National de la Recherche Scientifique, \\ Boîte Postale 166, 38042 Grenoble, France \\ and Institut National des Sciences Appliquées, 31077 Toulouse, France \\ J. Genoe ${ }^{\dagger}$ and D. K. Maude \\ High Magnetic Field Laboratory, Max-Planck-Institut für Festkörperforschung and Centre National de la Recherche Scientifique, \\ Boîte Postale 166, 38042 Grenoble, France \\ X. Kleber and L. B. Rigal \\ High Magnetic Field Laboratory, Max-Planck-Institut für Festkörperforschung and Centre National de la Recherche Scientifique, \\ Boîte Postale 166, 38042 Grenoble, France \\ and Institut National des Sciences Appliquées, 31077 Toulouse, France \\ Ulf Gennser \\ High Magnetic Field Laboratory, Max-Planck-Institut für Festkörperforschung and Centre National de la Recherche Scientifique, \\ Boîte Postale 166, 38042 Grenoble, France \\ J. C. Portal \\ High Magnetic Field Laboratory, Max-Planck-Institut für Festkörperforschung and Centre National de la Recherche Scientifique, \\ Boite Postale 166, 38042 Grenoble, France \\ and Institut National des Sciences Appliquées, 31077 Toulouse, France \\ K. E. Singer, P. Rutter, T. Taskin, A. R. Peaker, and A. C. Wright ${ }^{\S}$ \\ Department of Electrical Engineering and Electronics and Centre for Electronic Materials, University of Manchester Institute \\ of Science and Technology, Sackville Street, Manchester M601QD, United Kingdon \\ (Received 4 February 1997; revised manuscript received 28 April 1997)
}

\begin{abstract}
We have studied the magnetic properties of self-organized ErAs aggregates embedded in a GaAs matrix using telegraph noise spectroscopy. The electrical transport through the samples is mesoscopic and exhibits universal conductance fluctuations and two-level fluctuations (TLF's). A statistical analysis of a TLF allows us to measure the extremely small magnetization of an isolated ErAs cluster. Rotating the sample in a magnetic field reveals the sixfold magnetic anisotropy expected for ErAs with a rocksalt structure. At high temperatures the switching is thermally activated, while at low temperatures it is the tunneling of the magnetization which dominates. The magnetic-field dependence of the crossover temperature between the thermally activated and tunneling regimes can be explained with a simple model. [S0163-1829(98)06012-3]
\end{abstract}

\section{INTRODUCTION}

The tunneling of a macroscopic variable is a subject of considerable importance, since in some sense it forms a bridge between the quantum-mechanical and classical worlds. It has been suggested that magnetic systems should be good candidates to observe macroscopic quantum tunneling (MQT), ${ }^{1}$ A substantial amount of theoretical work has been performed to determine the low-temperature behavior of the switching of the magnetization. ${ }^{2}$ The reversal of the magnetization in small ferromagnetic ${ }^{1}$ or antiferromagnetic particles $^{3}$ has been considered together with the depinning of domain walls via a tunneling effect. ${ }^{4}$ This work suggests that antiferromagnetic materials are likely systems for the observation of the tunneling of the magnetization. Furthermore, the tunneling probability is increased when the size of the magnetic system is reduced. However, due to the experimental difficulties involved in measuring the extremely small magnetization of such systems, the experimental observation of MQT in a single isolated system is difficult.

Early work concentrated on large-scale samples where the depinning time of a large number of domain walls was studied. The observed saturation of the depinning time at low temperature was attributed to a noncoherent tunneling effect. ${ }^{5,6}$ Results on the tunneling of domain walls in ferromagnetic $^{7}$ or antiferromagnetic ${ }^{8}$ materials have also been reported. However, in these measurements a large number of domain walls switch simultaneously making the interpretation difficult. ${ }^{9}$ To overcome this problem, measurements have been carried out on individual elliptical ferromagnetic particles using a micro-dc superconducting quantum-interference device capable of measuring as few as $10^{5}$ electronic spins. ${ }^{10} \mathrm{~A}$ saturation of the magnetization reversal time was obtained at low temperatures, and attributed to the tunneling of the magnetization. ${ }^{11}$ Attempts to observe macroscopic quantum coherence have also been carried out 


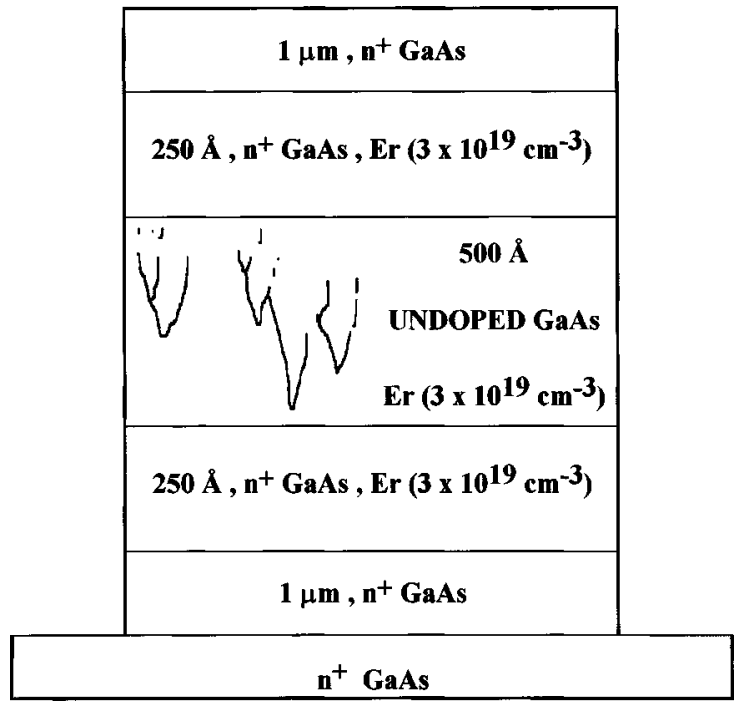

FIG. 1. Schematic representation of the sample structure.

using a microfabricated microsusceptometer. ${ }^{12}$

Recently, ${ }^{13}$ we used the mesoscopic electrical properties of self-organized ErAs precipitates in a GaAs matrix to observe MQT. A statistical analysis of two-level fluctuations (TLF's) appearing in the resistance was used to measure the small magnetization of an isolated antiferromagnetic ErAs cluster containing $\sim 1000$ atoms. The TLF in the resistance arises due to the coherent nature of the transport through the device. The resistance of the sample is sensitive to the scattering from a single cluster, which depends on the orientation of the magnetic moment of the cluster. If the orientation of the cluster is continuously switching between two possible orientations then this leads to a TLF in the resistance. Using this technique it is possible to measure extremely small magnetic moments-i.e., of the order of ten electronic spins. At low temperature, a saturation of the switching rate was observed and attributed to MQT.

This paper describes an extended investigation of the magnetic properties of self-organized ErAs clusters using telegraph noise spectroscopy. In Sec. II a detailed description of the sample is presented. The relation between the mesoscopic electrical properties of the sample and the magnetic properties, including the low-temperature magnetic phase diagram of the ErAs precipitates is discussed in Secs. III and IV. In Sec. V, in situ rotation measurements are presented, which allow us to probe the anisotropy of the magnetic potential of the ErAs clusters. A detailed discussion on the MQT is given in Sec. V. The magnetic-field dependence of the cross-over temperature between the thermally activated and tunneling regimes is explained using a simple model.

\section{SAMPLE DESCRIPTION AND EXPERIMENTAL DESCRIPTION}

Several GaAs $n-i-n$ structures with self-organized ErAs precipitates were grown using molecular-beam epitaxy. The growth sequence is as follows (see also Fig. 1): a 1- $\mu$ m-thick Se-doped $\left(N_{d}=10^{18} \mathrm{~cm}^{-3}\right)$ layer was grown on a (100) $n^{+}$ substrate, followed by a 25 -nm-thick GaAs layer with the same Se concentration but with an additional Er doping of $3 \times 10^{19} \mathrm{~cm}^{-3}$. $50 \mathrm{~nm}$ of GaAs $\mathrm{Er}$ (Er concentration 3

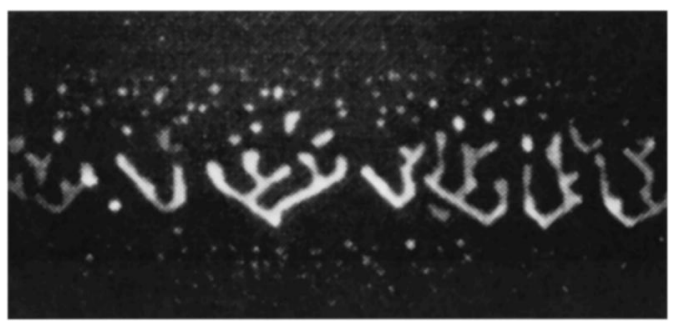

FIG. 2. Transmission electron microscope image of the ErAs quantum tree structure. The growth direction is upward and the size of the picture is $120 \times 250 \mathrm{~nm}^{2}$.

$\times 10^{19} \mathrm{~cm}^{-3}$ ) was grown with no intentional $n$-type doping. Identical GaAs:(Se,Er) and GaAs:Se layers were subsequently grown to form the top contact. Under the growth conditions used, ${ }^{14,15}$ the ErAs aggregates into very small interconnected quantum wires in the intrinsic regions and quantum dots in the Se-doped regions.

Cross-sectional transmission electron microscopy (see Fig. 2) shows that ErAs-wires and dots, embedded in the GaAs, have an average diameter of $3 \mathrm{~nm}$. While it is difficult to obtain information on the crystal structure of the dots and wires, a TEM investigation indicates the absence of dislocations in the GaAs host matrix. We therefore conclude that the ErAs is incorporated with a rocksalt structure, with the small lattice mismatch $(\sim 1.6 \%)$ being taken up elastically. Mesa structures, of size $10 \times 15 \mu \mathrm{m}^{2}$ were fabricated using conventional photolithography and wet etching techniques. Ohmic contacts were formed with alloyed AuGe/Ni.

For the transport measurements the sample was mounted on the rotation stage in a dilution refrigerator such that $0^{\circ}$ corresponds with the magnetic field aligned along the [001] axis, and $90^{\circ}$ with the field aligned along the [110] axis. All resistance measurements were performed in a local configuration using classical lock-in techniques and at temperatures between $50 \mathrm{mK}$ and $10 \mathrm{~K}$.

\section{UNIVERSAL FLUCTUATIONS CONDUCTANCE}

The samples show aperiodic oscillations in the magnetoresistance at low temperatures. ${ }^{13,16}$ These fluctuations are characteristic of universal conductance fluctuations (UCF's) in mesoscopic samples, ${ }^{17}$ and are due to interference of the partial carrier wave functions as they traverse the structure. From the typical period of the fluctuations, a phase coherence length of $L_{\phi}=100 \mathrm{~nm}$ can be deduced. The amplitude of the conductance fluctuations has been estimated to be $\Delta G=0.1 e^{2} / \mathrm{h}$.

A sample with all its dimensions lower than $L_{\phi}$ will exhibit fluctuations in the conductance with an amplitude of the order of $e^{2} / \mathrm{h}$ at zero $\mathrm{K} .{ }^{18}$ When the sample is larger than $L_{\phi}$, it can be divided into subsystems (of size $L_{\phi}$ ) in parallel and in series. Each subsystem will fluctuate with an amplitude of the order of $e^{2} / \mathrm{h}$. The total amplitude of the fluctuations can be determined by combining conductance fluctuating independently in series and parallel. ${ }^{17}$ Subsystems in parallel will increase the total amplitude of the fluctuation, while subsystems in series will decrease it. For a sample in which the current is propagating in the $z$ direction, and of sizes $L_{x}, L_{y}$, and $L_{z}$, the total amplitude of the fluctuations is described by ${ }^{17}$ 


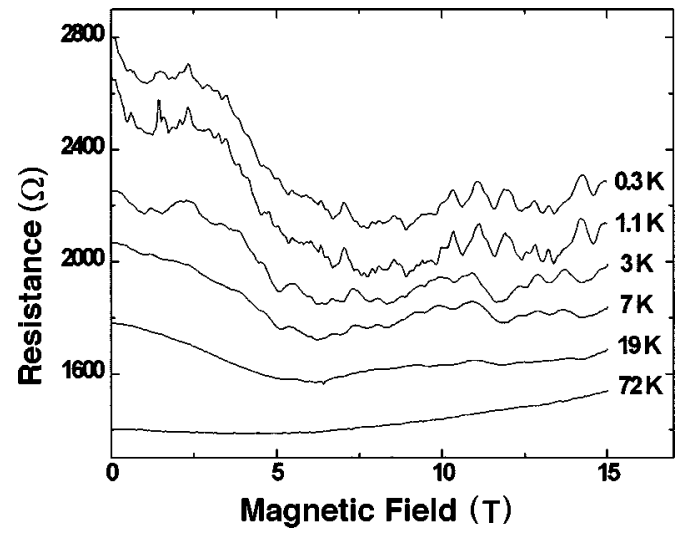

FIG. 3. Resistance of a $150-\mu \mathrm{m}^{2}$ mesa as a function of magnetic field for different temperatures. The curves have been shifted vertically for clarity.

$$
\Delta G=\frac{e^{2}}{\mathrm{~h}}\left(\frac{L_{x} L_{y}}{L_{\phi}^{2}}\right)^{1 / 2}\left(\frac{L_{\phi}}{L_{z}}\right)^{3 / 2},
$$

where $\left(L_{x} L_{y} / L_{\phi}^{2}\right)$ and $\left(L_{z} / L_{\phi}\right)$ are the number of subsystems in parallel and in series, respectively. This behavior has been observed experimentally in our sample by changing the size of the mesa (i.e., changing the number of subsystems in parallel). The amplitude of the fluctuations in the conductance was $0.1 e^{2} / \mathrm{h}, 0.5 e^{2} / \mathrm{h}$, and $3 e^{2} / \mathrm{h}$. for mesa sizes of 150,9500 , and $34000 \mu \mathrm{m}^{2}$, respectively. When the number of subsystems in parallel is increased, the amplitude of the fluctuations are increased.

Even for the smallest $\left(150 \mu \mathrm{m}^{2}\right)$ mesa the number of subsystems in parallel is expected to be large $\sim S / L_{\phi}^{2}=10^{6}$, where $S$ is the surface of the mesa. Conversely, the number of systems in series is small because the thickness of the active region of the sample is of the same order of $L_{\phi}$ (see Fig. 1). According to Eq. (1), the amplitude of the fluctuations should be $10^{3} e^{2} / \mathrm{h}$ to compare with the measured value of $0.1 e^{2} / \mathrm{h}$. This discrepancy suggests an inhomogeneous transport through the sample. The resistance of the sample is controlled by only few systems of lateral size $L_{\phi}$ in parallel, leading to a reduction in the amplitude of the fluctuations.

These characteristics are particularly important for the observation of telegraph noise (TLF's) in the resistance due to, for example, the switching of the orientation of the magnetic moment of an ErAs cluster between two possible alignments. If we consider $N$ subsystems in parallel, the total amplitude of the fluctuations will be $N^{1 / 2} e^{2} / \mathrm{h}$. If one of the subsystems exhibits a TLF, the amplitude of this switcher will be of the order of $1 / N^{1 / 2}$, so that the total amplitude of the fluctuation may be below the sensitivity of the measurement. Furthermore, the presence of a large number of subsystems in parallel would imply the presence of a large number of switchers, which would make the observation of a single TLF impossible.

\section{RELATION BETWEEN THE ELECTRICAL TRANSPORT AND THE MAGNETIC PROPERTIES OF ErAs}

A first hint as to the importance of the magnetic properties of ErAs for the electrical transport can be seen in Fig. 3. At

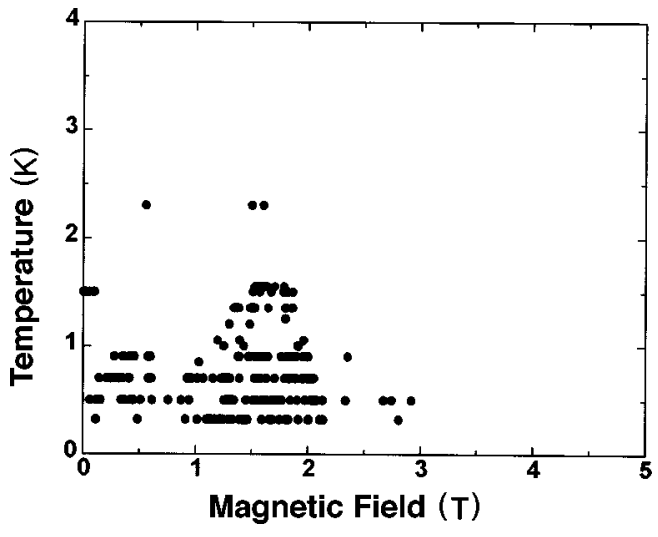

FIG. 4. Magnetic-field position of the jumps in the resistance as a function of temperature (taken from a large number of sweeps performed between numerous thermal cycles to room temperature).

low temperatures a large negative magnetoresistance is observed for magnetic fields below $5 \mathrm{~T}$, with a shoulder at about $2 \mathrm{~T}$. When the temperature is increased, both the shoulder and the negative magnetoresistance disappear. These curves are similar to the longitudinal magnetoresistance measurements made on thin film of ErAs by Allen et $a l .{ }^{19}$ The shoulder corresponds to a maximum in the spindisordered scattering at the antiferromagnetic-paramagnetic phase transition. The negative magnetoresistance is a consequence of a reduction of the spin disorder scattering when all the spins align with the magnetic field. The observation of a shoulder in the curves in Fig. 3 shows the presence of an antiferromagnetic phase at low magnetic field and low temperature in our structure.

Superimposed on the UCF background, instabilities (sudden jumps) in the resistance are sometimes observed which result from the switching of the alignment of the magnetic moment of an isolated ErAs cluster between different possible orientations. The amplitude of these instabilities is of the same order of magnitude of the aperiodic fluctuations of conductance $\left(\approx 0.1 e^{2} / \mathrm{h}\right)$, confirming that the transport through the sample is limited by few subsystems in parallel of lateral size $L_{\phi}$. The critical current paths through the sample are changed by thermal cycling to room temperature, which allows us to investigate different switchers. In Fig. 4 we plot the magnetic field at which instabilities have been observed during numerous magnetic-field sweeps, and after a number of thermal cycles to room temperature. The instabilities occur at magnetic fields $H<3 \mathrm{~T}$ and low temperatures $T<3 \mathrm{~K}$ suggesting that the switching only occurs in the antiferromagnetic region of the ErAs phase diagram. The critical magnetic field $H_{c} \sim 2 \mathrm{~T}$ implied by Fig. 4 is somewhat higher than the $H_{c} \sim 1 \mathrm{~T}$ reported in Ref. 19 but nevertheless consistent with the appearance of a shoulder at $\sim 2 \mathrm{~T}$ in our low temperature magnetoresistance curves. We speculate that the phase diagram for ErAs dots and wires is modified by the strain due to the lattice mismatch and also due to shape anisotropy.

\section{TWO-LEVEL FLUCTUATIONS}

We have previously shown that a TLF can be modeled by a two-well system separated by a barrier. In the thermally 
activated regime, the average time spent in the $i$ th state is described by:

$$
\tau_{i}=\tau_{0, i} \exp \left(\frac{E_{i}}{k T}\right),
$$

where $E_{i}$ is the height of the barrier to escape from state $i$. ErAs has been shown to align its magnetic moments in the (111) plane; the magnetic moments have a ferromagnetic coupling within this plane and an antiferromagnetic coupling between successive (111) planes. ${ }^{19,20}$ The magnetocrystalline anisotropy therefore has six degenerate minima separated by $60^{\circ}$ in a (111) plane in the absence of magnetic field. In general, in our experiment to observe a TLF (i.e., to have the two states close to energetic alignment) a magnetic field is needed. ${ }^{13}$ Therefore the magnetic potential cannot be described only by the magnetocrystalline anisotropy. Stress or shape anisotropy introduces a term of the order 2 in the magnetic potential, and will remove the degeneracy of the minima at $0 \mathrm{~T}$.

When the magnetic field is changed, the heights of the different barriers change and the angular positions of the minima are displaced. An exact solution of the height of the barrier as a function of magnetic field depends on the different anisotropy constants, which are not known in our case and would require numerical simulation. However for small variations of the magnetic field we can assume that the positions of the minima are fixed, and that the variation of the barrier with magnetic field is mainly due to the difference of orientation of the magnetic moment of the state and the virtual state in the barrier. Within this approximation, for small variations of the magnetic field, the height of the barrier evolves linearly:

$$
E_{i}=E_{i}\left(\mathbf{H}_{0}\right)+\left(\mathbf{m}_{i}-\mathbf{m}_{b}\right) \cdot\left(\mathbf{H}-\mathbf{H}_{0}\right)
$$

Where $\mathbf{m}_{i}$ and $\mathbf{m}_{b}$ are the magnetization vectors corresponding to the state $i$ and to the virtual state in the barrier, $\mathbf{H}_{0}$ is the magnetic field required to energetically align the states in each well and $E_{i}\left(\mathbf{H}_{0}\right)$ the corresponding barrier height.

The average time spent in one state is then described by:

$$
\tau_{i}=\tau_{0, i} \exp \left(\frac{E_{i}\left(H_{0}\right)+\left(m_{i}-m_{b}\right) \cdot\left(H-H_{0}\right)}{k T}\right) .
$$

Thus it is possible to determine from the temperature and magnetic-field dependence the difference of the projection of the magnetic moment between the state and the virtual state corresponding to the maximum of the barrier onto the magnetic field axis $\left(m_{i}-m_{b}\right)$. The perfect exponential dependence of the average time spent in one state with the magnetic field (see Ref. 13) shows the validity of the approximation that the variation of the position of the state and of the maximum of the barrier is negligible for the limited range of magnetic field of interest here. The typical values of $m_{i}-m_{b}$ found $\left(10 \mu_{B}-50 \mu_{B}\right)$ agree well with estimates of the magnetic moment due to the uncompensated spins at the surface of an antiferromagnetic ErAs cluster. A particular TLF is observed over a small magnetic-field range for which the two states are energetically quite close. For other magnetic fields one of the states is dominant, and switching does not occur on an experimentally realistic time scale. To avoid repetition and for more details, the reader is

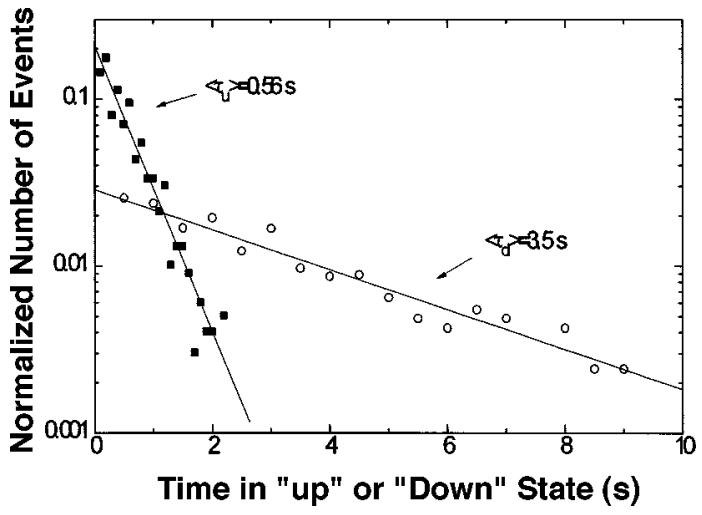

FIG. 5. Histogram of times spent in the high (closed squares) and low (open circles) states for TLF $c$ at $-1.53 \mathrm{~T}$ and $1.55 \mathrm{~K}$.

referred to Ref. 13. Here we adopt the same notation $(a-e)$ as in Ref. 13 for the different switchers investigated.

The Poisson nature of the statistics of the switching between states shows their uncorrelated nature. Figure 5 shows the cumulative distribution function of TLF $c$ at $-1.53 \mathrm{~T}$ and $1.55 \mathrm{~K}$. The slope of the exponential function corresponds to the average time, which is as a consequence a good estimator of the switching. For several TLF's (also in the tunneling regime) we have analyzed the stochastic nature of the switching by several techniques (autocorrelation function, power spectrum, histogram). They all confirmed the Poisson nature with one average time. This confirms the interpretation that only one cluster is switching.

By rotating the sample in the magnetic field, it is possible to extract information about the magnetic properties of the ErAs clusters. In particular the anisotropy of the magnetic potential can be probed and compared with the predicted anisotropy for ErAs with a rocksalt structure. The rotation of the sample is performed in situ in the dilution refrigerator as

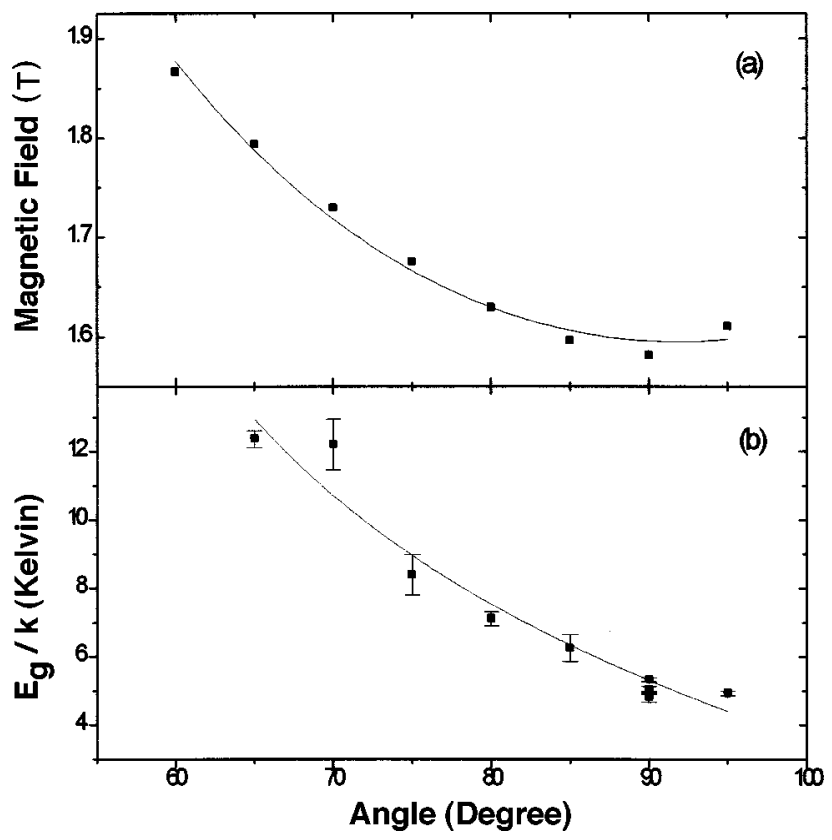

FIG. 6. Angular dependence of the magnetic field necessary to align the two states and the activation energy when the two states are aligned for TLF $e$. 


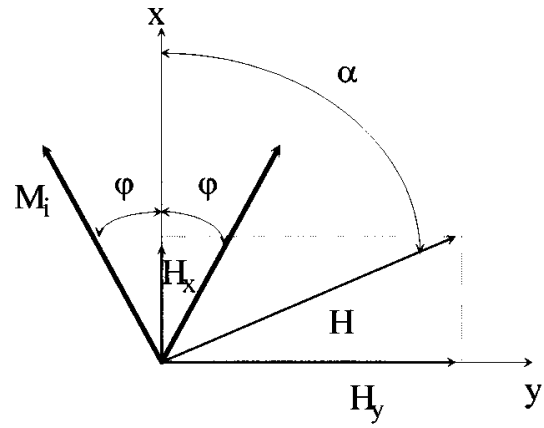

(a)

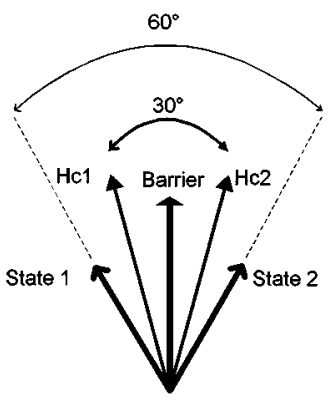

(b)

FIG. 7. (a) Schematic representation of the effect of the magnetic field on a TLF. $H_{x}$ allows us to align the two states, while $H_{y}$ changes the activation energy. (b) Position of the magnetic field ( $H c i)$ to obtain a value $\left(m_{i}-m_{b}\right)=0$ for state $i$. An angle of $30^{\circ}$ between the two magnetic-field directions corresponds to an angle of $60^{\circ}$ between the two states.

thermal cycling to room temperature changes the critical current paths through the sample and would result in the loss of the TLF under investigation.

First we demonstrate that by rotating the magnetic field it is possible to tune the barrier height and the energetic alignment of the two states. Figure 6 shows the dependence of the magnetic field required to energetically align ${ }^{21}$ the two states (a) and the height of the barrier when the two states are aligned (b) for different orientations of the magnetic field for TLF $e$. An angle of $0^{\circ}$ corresponds to a magnetic field along the [001] direction, and $90^{\circ}$ with the magnetic field along the [110] direction. The magnetic field required to aligned the two states decreases when the angle is increased, reaches a minimum at $90^{\circ}$ and starts to increase for angle greater than $90^{\circ}$. The activation energy (barrier height) decreases monotonically for all angles measured. An exact description of the problem would require a knowledge of the anisotropy constants and would be solvable only numerically. ${ }^{22}$ However, a good understanding of the behavior can be obtained by using two simple approximations. Noting the small range of magnetic field and angle used, we assume that the angular position of the states and of the maximum of the barrier do not change with the magnitude or orientation of the magnetic field. Under these assumptions, this behavior can be understood if we consider that the two states are separated by an angle $2 \varphi$ [see Fig. 7(a)] with the maximum of the barrier being in the middle of the two states. Once again we assume that the angular positions of the states are fixed, independent of the intensity and orientation of the magnetic field. The magnetic field can then be separated into two components

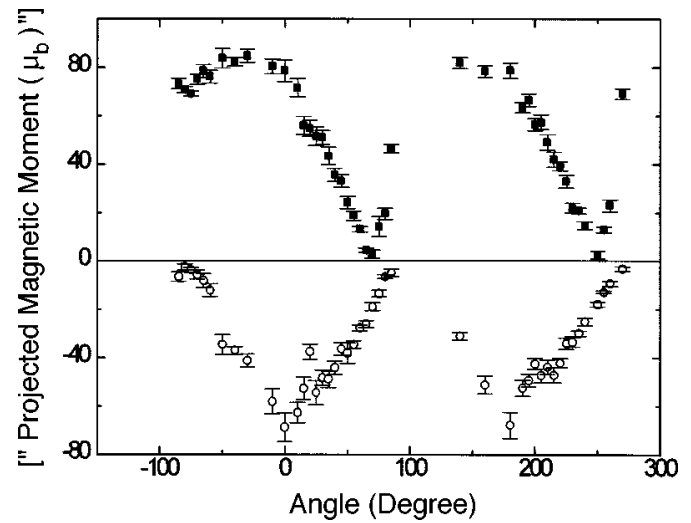

FIG. 8. Variation of the difference of magnetization projected onto the magnetic-field axis between states and the virtual state corresponding to the maximum of the barrier for TLF $g$.

$H_{x}=H \cos (\alpha)$ and $H_{y}=H \sin (\alpha)$, where $\alpha$ is the angle between the magnetic field and the maximum of the barrier. These two components have a very different effect on the system. $H_{x}$ will change the height of the barrier, but will have no effect on the difference in energy between the two states $\left(H_{x}\right.$ has the same effect on the two states). Only $H_{y}$ can change the relative position of the two states. Therefore, if $H_{y}^{*}$ is the magnetic field in the $y$ direction which is necessary to align the two states, the variation of the applied magnetic field required to align the two states $\left(H_{\text {alin }}\right)$ with the angle $\alpha$ is described by

$$
H_{\mathrm{alin}}=\frac{H_{y}^{*}}{\sin \alpha} .
$$

The magnetic field required to align the two states is a minimum when the magnetic field is perpendicular to the direction which bisects the two states. We note that within the approximation used here, when $\alpha=0$, an infinite magnetic field would be required to align the two states. In the real system this is of course not the case, as the position of the states (potential minima) would have to turn into the direction of the magnetic field. The variation with $\alpha$ of the height of the barrier is described by (assuming that the angular positions of the two states are fixed):

$$
E_{\text {act }}(\alpha) \propto \cot (\alpha) .
$$

The solid lines in Fig. 6 are fits of Eqs. (5) and (6) to the experimental points. Here we have assumed that the magnetic field is rotated in the plane of the two states. If this is not the case the analysis remains the same, except that only the projection of the magnetic field onto the plane should be considered.

For TLF $e$, for which a magnetic field of about 1.6 T was required to align the two states, it was only possible to follow the TLF over about $35^{\circ}$. In order to follow a switcher over a larger range, it is necessary to find a TLF which occurs at $H=0$, and therefore has no angular dependence for the magnetic field required to energetically align the two states. Such a switcher (TLF $g$ ) was found after a number of thermal cycles to room temperature. For each angle, the magnetic moment $\left(m_{i}-m_{b}\right)$ was measured for the low and high resistance states from a plot of $k T \ln \left(\left\langle\tau_{i}\right\rangle\right)$ versus mag- 
netic field. The results of the obtained magnetization as a function of the orientation of the external magnetic field are shown in Fig. 8. The value of $\left(m_{i}-m_{b}\right)$ has a somewhat sinusoidal variation as a function of angle with the zero values of $\left(m_{i}-m_{b}\right)$ for the high and low resistance states being shifted by $30^{\circ}$ with respect to each other. A value of $\left(m_{i}\right.$ $\left.-m_{b}\right)=0$ is obtained when the magnetic field lies along the direction which bisects the state $i$ and the virtual state in the barrier [see Fig. 7(b)]. Thus if the direction of the magnetic field for which $\left(m_{i}-m_{b}\right)=0$ for the two states is separated by $30^{\circ}$, the two states must be separated by $60^{\circ}$. This corresponds to the sixfold degeneracy of the magnetic potential observed in antiferromagnetic ErAs with a rocksalt structure. ${ }^{19,20}$ Not all the states are observed in the rotation experiment, probably due to the effect of strain or shape anisotropy, which favors two directions.

\section{TUNNELING OF MAGNETIZATION}

In Sec. $V$ we saw that by rotating the magnetic field it is possible to minimize the energy barrier separating the two states. Minimizing the energy barrier enhances the tunneling probability, a technique that we have used to observe the tunneling of the magnetization. ${ }^{13}$ In this section we discuss possible origins for the apparent deviation from detailed balance reported in Ref. 13. We also described a simple model to explain the evolution of the crossover temperature from the thermally activated behavior into the tunneling behavior as a function of magnetic field.

\section{A. Detailed balance}

Detailed balance (i.e., the dwell time in a state is proportional to the probability of being in that state) is universal, and must be obeyed in any two-level system,

$$
\frac{\left\langle\tau_{1}\right\rangle}{\left\langle\tau_{2}\right\rangle}=\frac{P_{1}}{P_{2}}=\exp \left(\frac{\varepsilon}{k T}\right),
$$

with $\left\langle\tau_{i}\right\rangle$ the dwell time on state $i, P_{i}$ the probability to be in state $i$, and $\varepsilon$ the difference in energy between both levels. This relation is valid in the thermally activated regime and in the tunneling regime and as a consequence also in the transition region between both. For the TLF's reported in Ref. 13 , this relation was not obeyed suggesting that the switcher cannot be treated as a simple two-level system. However, if we consider a two-well system with not only the ground state but with also different exited states populated, the situation is different. For example, if we consider two parabolic wells with different level spacing, Eq. (7) becomes

$$
\begin{aligned}
\frac{\left\langle\tau_{1}\right\rangle}{\left\langle\tau_{2}\right\rangle}= & \frac{\sinh \left(\frac{\Delta E_{2}}{2 k T}\right)}{\sinh \left(\frac{\Delta E_{1}}{2 k T}\right)} \exp \left(\frac{\varepsilon_{*}}{k T}\right) \\
& \approx \frac{\Delta E_{2}}{\Delta E_{1}} \exp \left(\frac{\varepsilon_{*}}{k T}\right) \quad \text { if both } \Delta E_{i} \ll k T,
\end{aligned}
$$

with $\Delta E_{i}$ the energy-level separation in well $i$, and $\varepsilon_{*}$ being energy separation between the bottoms of the two wells. The prefactor $\Delta E_{2} / \Delta E_{1}$ corresponds to a weighting of the ratio

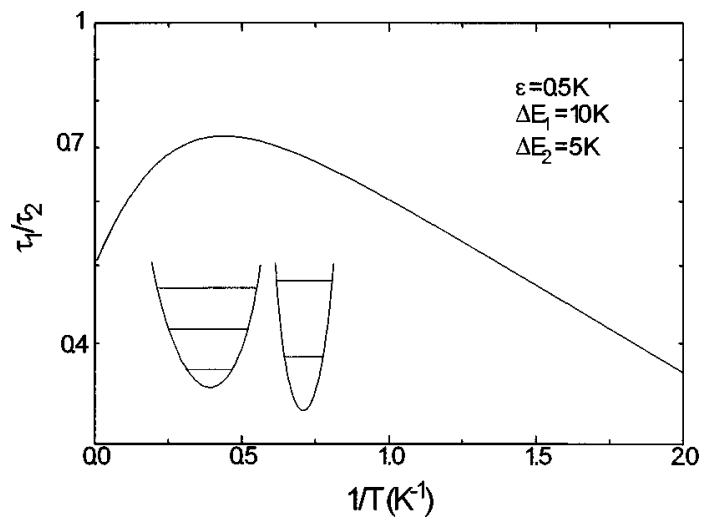

FIG. 9. Calculated detailed balance between two parabolic wells having a $0.5-\mathrm{K}$ energy difference between their lowest level, but with a doubled energy-level spacing in the higher well.

of the dwell times by the ratio of the density of states. Figure 9 shows the calculated variation of $\left\langle\tau_{1}\right\rangle /\left\langle\tau_{2}\right\rangle$ versus inverse temperature with the parameters indicated in the plot. The intercept in the high-temperature limit gives $\Delta E_{2} / \Delta E_{1}$, while the slope is determined by the energy difference between the bottom of each well. For low temperatures, however, the excited states are not populated and the system is well approximated by a simple two-level system, with an extrapolated high-temperature intercept of 1 and a slope determined by the energy separation of the groundstate in each well.

Obviously such a simple model is inadequate to explain the evolution of $\left\langle\tau_{1}\right\rangle /\left\langle\tau_{2}\right\rangle$ in our system which will depend on the exact form of the potential and the real density of states for the excited levels. Nevertheless, it shows the importance of the density of states in the shape of the curve $\left\langle\tau_{1}\right\rangle /\left\langle\tau_{2}\right\rangle$ as a function of the inverse temperature, and allows us to draw some qualitative understanding. The value where the high temperature slope crosses the zero of the $1 / T$ axis in the inset of Fig. 4 from Ref. 13, indicates that the ratio $\Delta E_{2} / \Delta E_{1}=2$ for TLF $e$. This also explains the difference in dwell times observed in the tunneling regime, even though the magnetic field was chosen such that the dwell times in the thermally activated regime were identical (see Fig. 4 in Ref. 13). Identical dwell times in the thermally activated regime do not imply that the two ground states are aligned. The factor of 2 in the energy spacing leads to a misalignment of the two wells equal to $\varepsilon_{*}$ $=k T \ln \left(\Delta E_{1} / \Delta E_{2}\right) \approx 0.7 k T$ (where $T$ is the temperature at which the two dwell times are equal). This corresponds to a misalignment of the ground state in each well of $\varepsilon=\varepsilon_{*}$ $-\left(\Delta E_{2}-\Delta E_{1}\right) / 2$, which results in the different dwell times observed in the tunneling regime. The sparse data in the tunneling regime prevents us from pursuing this analysis further. Further work to investigate the problem of detailed balance is underway.

It is clear from the data in Ref. 13 that the tunneling process is observed from the moment that the temperature is too low to populate the higher-energy levels significantly. This is perhaps not surprising as the mechanism populating the higher-energy levels and the thermally activated process are basically identical. For temperatures for which there is a significant thermal population of the excited states, thermally activated switching dominates over the tunneling process. 


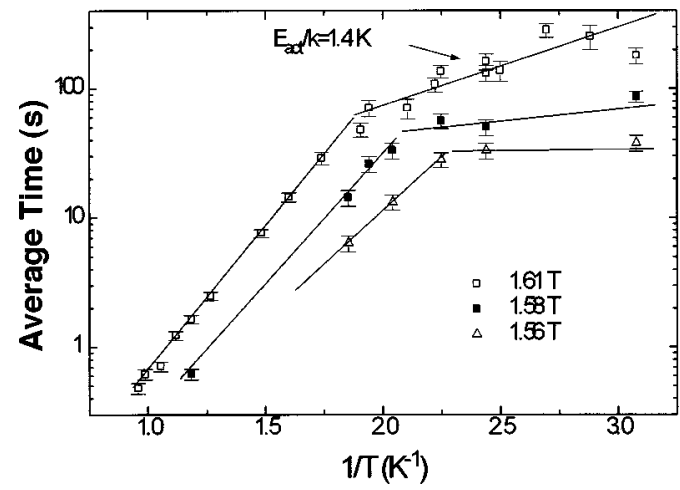

FIG. 10. Temperature dependence of the average time in the low state for TLF $e$ with different magnetic fields applied (1.61, 1.58, and $1.56 \mathrm{~T}$, respectively).

\section{B. Crossover temperature from \\ thermally activated behavior to quantum tunneling of magnetization}

In this section we investigate the variation with magnetic field of the crossover temperature from thermally activated behavior to quantum tunneling. Figure 10 shows the average time spent in the low state as a function of the inverse of temperature at three different magnetic fields (different bias) for TLF $e$. At high temperature the average time is thermally activated over two orders of magnitude. A saturation of the average dwell time due to tunneling of magnetization is observed at low temperature. At $1.56 \mathrm{~T}$, the ground states of each well are aligned, and the tunneling rate is temperature independent. Increasing the magnetic field biases the system, with the well corresponding to the lower resistance state moving to lower energy. This leads to thermally activated tunneling and the slope of $1.4 \mathrm{~K}$ in the tunneling regime at $1.61 \mathrm{~T}$ corresponds to the energy difference between the ground state in each well.

In addition, we observe a clear shift toward higher temperature of the crossover temperature when the magnetic field is increased. A similar behavior is observed for TLF $d$. The crossover temperature as a function of magnetic field is represented for these two TLF's in Fig. 11. In the thermally activated regime the average time $\left\langle\tau_{i}\right\rangle$ is described by Eq. (2). In the tunneling regime the average time is equal to

$$
\left\langle\tau_{i}\right\rangle=\tau_{0, i} \exp (\beta),
$$

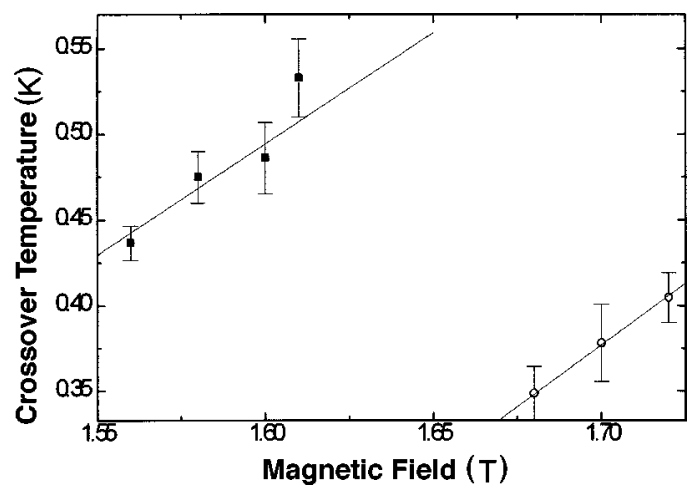

FIG. 11. The measured crossover temperature for TLF's $e$ and $d$. The solid lines give the predicted evolution according to the model in the text.

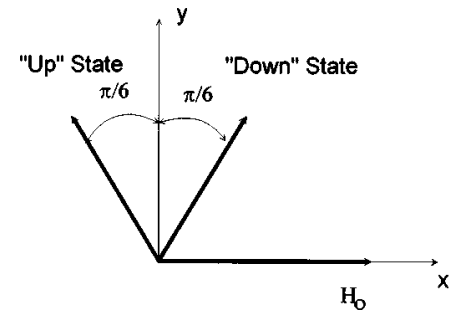

FIG. 12. Schematic representation of the configuration of the measurement. The magnetic field is perpendicular to the line which bisects the two states. Both states have an angle of $60^{\circ}$ between them.

where $\beta$ is the WKB coefficient. The crossover temperature $T^{*}$ is the temperature for which the tunneling rate is equal to the thermally activated rate, which occurs when

$$
\frac{E_{i}}{k T^{*}}=\beta
$$

The measurements presented in Fig. 11 were performed at an angle of $95^{\circ}$, which corresponded to the minimum of the barrier height. As we have already seen in Sec. V, the magnetic field required to align the two states is a minimum when the magnetic field is perpendicular to the maximum of the barrier. Furthermore, due to the sixfold symmetry of the magnetic potential, we assume that the two states are separated by an angle of $60^{\circ}$. This configuration is illustrated in Fig. 12. The variation of the barrier with magnetic field can be expressed as

$$
E_{i}=E_{0}+M_{1}\left(H-H_{0}\right),
$$

where $M_{1}$ is the projection of the low resistance state magnetization onto the magnetic field, and $E_{0}$ is the barrier height when a magnetic field $H_{0}$ is applied to energetically align the two states.

The variation of the WKB coefficient with magnetic field is difficult to predict as it would require a knowledge of the different anisotropy terms. In the literature, theoretical estimates of the WKB coefficient have been obtained for different ideal configurations. ${ }^{1,2,23}$ In the case of the reversal of the magnetization in an antiferromagnetic grain in the absence of magnetic field, the WKB coefficient has been shown to be $\mathrm{e}^{3,24}$

$$
\beta=\frac{\hbar \sqrt{\chi_{\perp} K}}{\mu_{b}} V=\frac{\hbar V^{1 / 2}}{\mu_{b}} \sqrt{E_{i}},
$$

where $K$ is the anisotropy constant and $\chi_{\perp}$ the perpendicular susceptibility and $V$ the volume of the grain. $E_{i}=K V$ is the height of the barrier. Therefore, in our measurement, if we assume that the general shape of the barrier does not change with the magnetic field (e.g., only the height of the barrier changes but not the position of the minimum) the WKB coefficient evolves as $\beta=C \sqrt{E_{i}}$ where $C$ is a constant. The assumption that the general shape of the barrier does not change with the magnetic field is confirmed by the linear variation of the barrier height with magnetic field. When the two states are aligned, $\beta=\beta_{0}$ and $E_{i}=E_{0}$, thus $C$ $=\beta_{0} / \sqrt{E_{0}}$. Therefore using Eqs. (10)-(14) the crossover temperature can be expressed as 


$$
\begin{aligned}
T^{*}(H) & =\frac{E_{0}}{k \beta_{0}}\left(1+\frac{M_{1}\left(H-H_{0}\right)}{E_{0}}\right)^{1 / 2} \\
& \approx \frac{E_{0}}{k \beta_{0}}\left(1+\frac{M_{1}\left(H-H_{0}\right)}{2 E_{0}}\right) .
\end{aligned}
$$

All the parameters in this equation can be determined independently from the measurement of the dwell time as a function of the temperature when the two states are aligned. The slopes in Fig. 12 are calculating using this simple model, and we observe an excellent correspondence with the experimental values of the crossover temperatures. We note that the increase of the crossover temperature with magnetic field is consistent with existing theory in different configurations, where the crossover temperature increases when the height of the barrier decreases. ${ }^{2,24}$

\section{CONCLUSION}

We have studied the electrical transport of self-organized ErAs aggregates in a GaAs matrix. Telegraph noise or TLF's are observed in the resistance of the sample and correspond to the switching of the magnetization of an ErAs cluster between two possible orientations. The statistical analysis of the TLF allows us to measure the extremely small magnetization carried by a cluster of the order of only few Bohr magnetons. Rotating the sample in situ provides evidence of the sixfold degeneracy of the magnetocrystalline anisotropy of ErAs. Previous work on the tunneling of the magnetization has been extended. In particular we have shown the importance of the excited states of the system, which allows us to understand the apparent deviation from detailed balance. Finally, the dependence of the crossover temperature between the thermally activated and the tunneling regimes as a function of the magnetic field (well bias) is in good agreement with a simple theoretical model.

\section{ACKNOWLEDGMENTS}

We thank B. Barbara, N. Birge, H. B. Braun, P. Stamp, and Y. De Wilde for fruitful discussions. This research was supported by the E.U. through ESPRIT BRA III Contract No. 7260. J.G. acknowledges the Human Capital and Mobility Program of the E.U. for financial support.
*Present address: Dept. of Electrical Engineering, UCLA, Los Angeles, CA 90095-1594.

Present address: ETRO, VUB, Pleinlaan 2, B-1050 Brussels, Belgium and MAP-MBE, IMEC, Kapeldreef 75, B-3001 Leuven, Belgium.

${ }^{\ddagger}$ Present address: Paul Scherrer Institut, Villigen, Switzerland.

${ }^{\S}$ Also at AMRL, N.E. Wales Institute, Deeside College, Connah's Quay, CH5 4HR, Wales, United Kingdom.

${ }^{1}$ E. M. Chudnovsky and L. Gunther, Phys. Rev. Lett. 60, 661 (1988).

${ }^{2}$ For a review, see P. C. E. Stamp, E. M. Chudnovsky, and B. Barbara, Int. J. Mod. Phys. B 6, 1355 (1992).

${ }^{3}$ B. Barbara and E. M. Chudnovsky, Phys. Rev. A 145, 205 (1990).

${ }^{4}$ P. C. E. Stamp, Phys. Rev. Lett. 66, 2802 (1991).

${ }^{5}$ M. Uehara and B. Barbara, J. Phys. (Paris) 47, 235 (1986).

${ }^{6}$ B. Barbara, P. C. E. Stamp, and M. Uehara, J. Phys. (Paris) 49, C8 (1988).

${ }^{7}$ C. Paulsen, L. C. Sampaio, B. Barbara, R. Tucoulou-Tachoueres, D. Fruchart, A. Marchand, J. L. Tholence, and M. Uehara, Europhys. Lett. 19, 643 (1992).

${ }^{8}$ J. Tejada, X. X. Zhang, A. Roig, O. Nikolov, and E. Molins, Europhys. Lett. 30, 227 (1995).

${ }^{9}$ B. Barbara and L. Gunther, J. Magn. Magn. Mater. 128, 35 (1993).

${ }^{10}$ W. Wernsdorfer, K. Hasselbach, D. Mailly, B. Barbara, A. Benoit, L. Thomas, and G. Suran, J. Magn. Magn. Mater. 145, 33 (1995).

${ }^{11}$ B. Barbara, W. Wernsdorfer, L. C. Sampaoi, J. G. Park, C. Paulsen, M. A. Novak, R. Ferré, D. Mailly, R. Sessoli, A. Can- eschi, K. Hasselbach, A. Benoit, and L. Thomas, J. Magn. Magn. Mater. 140-144, 1825 (1995).

${ }^{12}$ D. D. Awschalom, M. A. McCord, and G. Grinstein, Phys. Rev. Lett. 65, 783 (1990).

${ }^{13}$ F. Coppinger, J. Genoe, D. K. Maude, Ulf Gennser, J. C. Portal, K. E. Singer, P. Rutter, T. Taskin, A. R. Peaker, and A. C. Wrigth, Phys. Rev. Lett. 75, 3513 (1995).

${ }^{14}$ I. Poole, K. E. Singer, A. R. Peaker, and A. C. Wright, J. Cryst. Growth 121, 121 (1992).

${ }^{15}$ K. E. Singer, P. Rutter, A. R. Peaker, and A. C. Wright, Appl. Phys. Lett. 64, 707 (1994).

${ }^{16}$ F. Coppinger, D. K. Maude, Ulf Gennser, J. C. Portal, K. E. Singer, P. Rutter, A. R. Peaker, and A. C. Wrigth, Proceedings of ICPS-22 (World Scientific, Singapore, 1995), Vol. 2, p. 1791.

${ }^{17}$ See, for example, P. A. Lee, A. Douglas Stone, and H. Fukuyama, Phys. Rev. B 35, 1039 (1987).

${ }^{18}$ P. A. Lee and A. Douglas Stone, Phys. Rev. Lett. 55, 1622 (1985).

${ }^{19}$ S. J. Allen, Jr., N. Tabatabaie, C. J. Palmstrom, G. W. Hull, T. Sands, F. de Rosa, H. L. Gilchrist, and K. C. Garrison, Phys. Rev. Lett. 62, 2309 (1989).

${ }^{20}$ H. R. Child, M. K. Wilkinson, J. W. Cable, W. C. Koehler, and E. O. Wollan, Phys. Rev. 133, 922 (1963).

${ }^{21}$ Here we assume that the states are aligned when the average time spent in each state is identical. This is in fact an oversimplification - see the discussion on detailed balance concerning this point.

${ }^{22}$ A. H. Morrish, The Physical Principles of Magnetism (Wiley, New York, 1965).

${ }^{23}$ A. Garg and G. H. Kim, J. Appl. Phys. 67, 5669 (1990).

${ }^{24}$ E. G. Chudnovsky, J. Magn. Magn. Mater. 140-144, 1821 (1995). 